() М.В.Шестакова ${ }^{1}$, М.Б. Анциферов ${ }^{2}$, А.С. Аметов ${ }^{3}$, Г.Р.Галстян ${ }^{1}$, Т.Ю. Демидова ${ }^{4}$, А.В. Зилов ${ }^{5}$, Т.Н. Маркова ${ }^{6,7}$, Н.А. Петунина ${ }^{5}$, Н.А. Черникова ${ }^{3}$, М.Ш. Шамхалова'

1Национальный медицинский исследовательский центр эндокринологии, Москва

Эндокринологический диспансер Департамента здравоохранения города Москвы, Москва

${ }^{3}$ Российская медицинская академия непрерывного профессионального образования, Москва

${ }^{4}$ Российский национальный исследовательский медицинский университет имени Н.И. Пирогова, Москва

5 Первый Московский государственный медицинский университет им. И.М. Сеченова (Сеченовский Университет),

Москва

бГродская клиническая больница №52, Москва

${ }^{7}$ Московский государственный медико-стоматологический университет им. А.И. Евдокимова, Москва

По данным ключевых диабетологических исследований, раннее назначение сахароснижающей терапии метформином сопровождается снижением риска развития микро- и, в перспективе длительного 10-летнего наблюдения, макрососудистых осложнений и сердечно-сосудистой смертности. Результаты краткосрочных исследований комбинированной сахароснижающей терапии метформином предполагают, что комбинированная терапия может иметь ряд преимуществ как с позиций эффективности гликемического контроля, так и с позиций положительного воздействия на развитие осложнений сахарного диабета 2 типа (СД2). Остается открытым вопрос времени старта комбинированной сахароснижающей терапии. По результатам недавних широкомасштабных исследований, данных реальной клинической практики, тщательный гликемический контроль в течение первого года с момента постановки диагноза СД2 имеет решающее значение для дальнейшего управления заболеванием и замедления прогрессирования осложнений.

Однако в силу того, что клинические преимущества ранней комбинированной терапии не были продемонстрированы в рандомизированных клинических исследованиях (РКИ), такой подход, несмотря на теоретические предпосылки, не был рекомендован к широкому применению в международных руководствах по лечению пациентов с СД2. Российские алгоритмы оказания специализированной помощи больным сахарным диабетом рекомендуют комбинированную сахароснижающую терапию на старте лечения при уровне $\mathrm{Hb}_{1 с}$ выше целевого на $1 \%$.

В 5-летнем исследовании VERIFY ((Vildagliptin Efficacy in combination with metfoRmln For earlY treatment of type 2 diabetes) продемонстрированы длительный устойчивый контроль гликемии на фоне комбинированной терапии вилдаглиптин+метформин, назначенной пациентам с впервые установленным СД2 при относительно низких значениях $\mathrm{HbA}_{1 c^{\prime}}$ а также преимущества данного подхода в сравнении со стандартной стратегией поэтапной интенсификации монотерапии. Результаты исследования VERIFY предоставили большое количество информации для обсуждения вопросов ранней интенсификации лечения, клинических преимуществ такого подхода и возможного пересмотра стратегии лечения пациентов с впервые установленным диагнозом СД2.

КЛЮЧЕВЫЕ СЛОВА: сахарный диабет; вилдаглиптин; метформин; $Н b A_{1 ;}$ в впервые выявленный СД

\title{
WHAT ARE NEW OPPORTUNITIES FOR CLINICAL PRACTICE THE VERIFY STUDY OPENS AND WHICH VALUES FOR NATIVE DIABETES PATIENTS? JOINT CONCLUSION ON THE ADVISORY BOARD RESULTS. NOVEMBER 6, 2019
}

(c) Marina V. Shestakova', Michail B. Antsiferov², Alexander S. Ametov ${ }^{3}$, Gagik R. Galstyan' ${ }^{1}$, Tatiana Y. Demidova ${ }^{5}$, Alexey V. Zilov', Tatyana N. Markova ${ }^{6,7}$, Nina A. Petunina ${ }^{5}$, Natalya A. Chernikova ${ }^{3}$, Minara S. Shamkhalova ${ }^{1}$

'Endocrinology Research Centre, Moscow, Russia

${ }^{2}$ Endocrinology department of the Moscow Health Department, Moscow, Russia

${ }^{3}$ Russian Medical Academy of Continuous Professional Education, Moscow, Russia

${ }^{4}$ Pirogov Russian National Research Medical University, Moscow, Russia

${ }^{5}$ I.M. Sechenov First Moscow State Medical University (Sechenov University), Moscow, Russia

${ }^{6}$ City Clinical Hospital No52, Moscow, Russia

${ }^{7}$ A.I. Yevdokimov Moscow State University of Medicine and Dentistry, Moscow, Russia 
According to key diabetic studies, the early use of metformin glucose lowering therapy is associated with a reduced risk of developing micro- and, in the long term, 10-year follow-up, macrovascular complications and cardiovascular mortality. Short-term studies results on combined glucose lowering therapy with metformin suggests that combination therapy can have several advantages on the one side from the effectiveness of glycemic control and on another side from positive effect on the development of complications of type 2 diabetes. The question of the start time of combined hypoglycemic therapy remains open. According to the results of recent large-scale studies, real world evidence data, careful glycemic control during the first year from the moment of diagnosis of type 2 diabetes is crucial for further management of the disease and slow the progression of complications.

However, due to the fact that the clinical benefits of early combination therapy were not demonstrated in randomized clinical trials, this approach, despite the theoretical background, was not recommended for widespread use in international guidelines for the treatment diabetes patients. Russian algorithms on the treatment diabetes patients recommend combined glucose lowering therapy at the start of treatment at a $\mathrm{HbA} 1 \mathrm{c}$ level of $1 \%$ higher than the target.

A 5-year VERIFY study results were demonstrated long-term sustained glycemic control in combination with vildagliptin + metformin prescribed for native diabetes patients with relatively low $\mathrm{HbA}_{1 \mathrm{c}}$ values, as well as the advantages of this approach in comparison with the standard strategy for phased intensification of monotherapy. The results of the VERIFY study provided a wealth of information to discuss early treatment intensification, the clinical benefits of this approach and a possible review of the treatment strategy for native diabetes patients.

KEYWORDS: diabetes mellitus; vildagliptin; metformin; $\mathrm{HbA}_{1 c^{\prime}}$ new onset diabetes mellitus

\section{ЦЕЛИ И ЗАДАЧИ СОВЕТА ЭКСПЕРТОВ}

Основная цель заседания - обсудить преимущества стратегии ранней комбинированной терапии на старте лечения СД2 с точки зрения патофизиологии заболевания, целей и задач гликемического контроля, преимуществ для пациентов и клинических исходов лечения, а также разработать алгоритм стартовой комбинированной терапии СД2.

\section{РЕЗОЛЮЦИЯ СОВЕТА ЭКСПЕРТОВ}

СД2 является мультипатогенетическим заболеванием с многочисленными отклонениями. В настоящее время известно 11 дефектов, участвующих в развитии и прогрессировании заболевания. Вторая особенность СД2 заключается в том, что в момент дебюта все патологические механизмы уже запущены и далеко продвинулись в своем воздействии на органы, системы и организм в целом. Результатом является тот факт, что на протяжении многих лет у пациентов существует неконтролируемая глюкотоксичность, которая начинает действовать достаточно рано, как только превышается порог физиологических значений, создавая в итоге отрицательный метаболический фундамент. И, как правило, лечение начинается тогда, когда отрицательный метаболический фундамент уже сформировался. Все осложнения являются результатом множества повторяющихся процессов на протяжении всего заболевания. Драйвером осложнений являются те же воздействия и процессы, которые повреждают $\beta$-клетки: избыточная продукция глюкозы печенью и гиперглюкагонемия, снижение утилизации глюкозы на уровне клеток-мишеней, повышение уровня триглицеридов и свободных жирных кислот, оксидативный стресс, хроническая гиперактивация иммунной системы метаболическим стрессом, иммунологическое воспаление, что в совокупности поддерживает и способствует нарастанию глюкотоксичности и повреждению $\beta$-клеток. Современная концепция патофизиологии СД2 рассматривает 11 патогенетических дефектов, которые объединены в $\beta$-ориентированную модель, подчеркивая, что именно дисфункция $\beta$-клеток является ведущим нарушением в развитии и прогрессировании заболевания [1]. На основании вышеизложенного сформировалась концепция патофизиологического подхода: для коррекции множественных патологических дефектов необходима комбинация препаратов, лечение должно основываться на коррекции патогенетических аномалий, а не просто на снижении уровня $\mathrm{HbA}_{1 c^{\prime}}$ терапия должна быть начата рано для предотвращения/замедления прогрессирующей дисфункции $\beta$-клеток [2].

Дисфункция $\beta$-клеток обратима до определенного этапа, далее начинают включаться процессы, ведущие к потере популяционной идентичности и трансдифференциации, что в дальнейшем приводит к гибели $\beta$-клеток. Если $\beta$-клетки находятся в состоянии покоя как дедифференцированные клетки, теоретически есть возможность восстановить их активность. И сделать это важно как можно раньше, т.к. вместе со снижением массы функционирующих $\beta$-клеток снижается инкретиновый эффект. В норме вклад «инкретинового эффекта» в уровень постпрандиального инсулина составляет около 70\%, что имеет огромное клиническое значение, т.к. снижение «инкретинового эффекта» регистрируется уже со стадии нарушения толерантности к глюкозе [3, 4]. Сахароснижающие препараты, направленные на сохранение или даже увеличение функции $\beta$-клеток, являются перспективным и оправданным подходом к долгосрочному контролю уровня гликемии. Ингибиторы дипептидилпептидазы-4 (иДПП-4), действуя глюкозозависимо, способствуют улучшению функции $\beta$-клеток преимущественно благодаря повышению чувствительности к глюкозе [5, 6], независимо от степени снижения гипергликемии $[7,8]$. Ингибирование ДПП-4 усиливает чувствительность $\beta$-клеток к глюкозе в большом диапазоне концентраций глюкозы (от гипогликемических до гипергликемических). Благодаря этому $\beta$-клетки могут адаптироваться к имеющейся степени инсулинорезистентности, что способствует большей степени коррекции гликемии натощак и после приема пищи $[9,10]$. Смена парадигмы стартовой терапии СД2 
на «ß-центрическую» будет способствовать более полной, обоснованной и продолжительной терапии пациентов с СД2, в то время как глюкоцентрическая концепция рассматривает пациентов с ранними проявлениями нарушения углеводного обмена, т.е. пациентов с невысоким уровнем гликемии, как имеющих менее тяжелые дефекты, таким образом как бы оправдывая отсрочку интенсификации терапии [11]. Таким образом, терапия, направленная на замедление прогрессирования дисфункции $\beta$-клеток, является оправданной с точки зрения патофизиологии заболевания, потенциал такой терапии максимально реализуется на ранних этапах развития заболевания.

В одном из ключевых исследований в области лечения СД2 - UKPDS - было доказано, что хороший гликемический контроль ассоциирован со снижением рисков микрососудистых осложнений. По окончании 10 лет исследования разница по показателю $\mathrm{HbA}_{1 с}$ составила 0,9\% между группами интенсивной и традиционной сахароснижающей терапии, что сопровождалось снижением риска развития микрососудистых осложнений в группе интенсивной терапии на 25\% [12]. В течение дальнейшего 10-летнего наблюдения уровни $\mathrm{HbA}_{1 c}$ в группах сравнялись, но риски микрососудистых осложнений по-прежнему были меньше в группе интенсивной терапии в сравнении с традиционной. Далее, по окончании еще 10 лет наблюдения, было получено статистически значимое снижение рисков развития макрососудистых осложнений: инфаркта миокарда на 15\% и общей смертности на 13\% [13]. На основании этих данных была сформулирована концепция «метаболической памяти», которая акцентирует необходимость ранней интенсивной сахароснижающей терапии для снижения рисков развития микро- и макрососудистых осложнений СД 2 типа с целью первичной профилактики неблагоприятных сердечно-сосудистых исходов. Публикация данных UKPDS и пересмотр алгоритмов лечения на основании результатов UKPDS, в частности включение рекомендации по строгому контролю гликемии, привело к снижению частоты осложнений, связанных с СД2 [14]. В анализе широкомасштабного исследования реальной клинической практики - Diabetes \& Aging Study, включавшем около 28 тыс. пациентов с впервые установленным СД2, было показано, что нестабильный контроль и, соответственно, профили $\mathrm{HbA}_{1 c}$ связаны с большим риском неблагоприятных исходов в сравнении с низким стабильным удержанием $\mathrm{HbA}_{1 c}$ в диапазоне около 7\% [15]. В последующем анализе этого исследования на расширенных данных 35 тыс. пациентов с впервые установленным СД2 установлено, что достижение и стабильное удержание целевых уровней гликемии в течение каждого последующего года с момента постановки диагноза позволяет не только снизить риск развития осложнений СД2, включая смертельные исходы, но и сохранить качество жизни указанных больных в 10-летней перспективе, а также была подчеркнута важность стабильного контроля гликемии, начиная со старта лечения, т.к. уже в течение первого года недостижения гликемического контроля отмечается повышение риска осложнений [16]. Таким образом, поддержание устойчивого гликемического контроля, особенно в первые годы терапии после дебюта заболевания, является залогом успеха лечения пациента в це- лом, снижая риск развития осложнений и неблагоприятных сердечно-сосудистых исходов.

Следуя положениям патофизиологического подхода, на старте СД2 должна назначаться комбинированная терапия с целью большей степени коррекции, в первую очередь множественных патогенетических нарушений. Комбинация метформина с иДПП-4 является комплементарной по механизму действия препаратов, т.к. воздействует на основные звенья патогенеза СД 2 типа: дисфункцию $\beta$-клеток и инсулинорезистентность. Другие варианты стартовой комбинированной терапии также применимы и оправданы с точки зрения патогенетической терапии, возможно, старт с трех препаратов будет еще лучше с позиции воздействия на большее число факторов патогенеза. Несмотря на имеющиеся сведения о пользе ранней комбинированной терапии, обусловленной патофизиологической направленностью механизмов действия и более выраженным начальным снижением уровня гликемии по сравнению с монотерапией метформином [17], данных, подтверждающих преимущества стратегии ранней комбинированной терапии в сравнении со стратегией поэтапной интенсификации, было недостаточно до оглашения результатов исследования VERIFY. Исследование VERIFY - первое исследование долгосрочных клинических преимуществ стратегии ранней комбинированной терапии. Благодаря прогрессу в понимании патофизиологических механизмов, лежащих в основе прогрессирования СД 2-го типа, и расширению возможностей лечения, терапия, рассматривавшаяся в UKPDS в качестве интенсивной, использовалась в исследовании VERIFY в качестве сравнения. В исследовании VERIFY сравнивалась стратегия ранней комбинированной терапии со стратегией поэтапной интенсификации монотерапии метформином у пациентов с впервые установленным СД2 (длительность СД2 в среднем 3,3 мес) с относительно низким уровнем $\mathrm{HbA}_{1 c}$ (среднее значение 6,7\%) и глюкозы плазмы натощак (среднее значение 6,9 ммоль/л). Сравнение проводилось по времени удержания гликемического контроля (потерей контроля считалось увеличение $\mathrm{HbA}_{1 с}$ более $7 \%$ по 2 последовательным измерениям через 3 мес каждое).

Исследование VERIFY показало преимущества стратегии ранней комбинированной терапии «вилдаглиптин плюс метформин» для поддержания длительного устойчивого уровня гликемии в течение более 5 лет в сравнении со стратегией поэтапной интенсификации монотерапии метформином: вероятность ускользания контроля ниже на 49\%, в среднем на два года дольше удержание стабильного контроля (61,9 мес в группе ранней комбинированной терапии и 36,1 мес в группе монотерапии метформином), вероятность необходимости назначения третьего препарата ниже на 26\% [18]. Дополнительная ценность полученных результатов заключается в том, что сравнение проводилось с оптимальной на момент старта исследования стратегией интенсификации: второй препарат назначался сразу же при превышении уровня $\mathrm{HbA}_{1 c}$ 7\%, и даже при таком сравнении вероятность удержания стабильного гликемического контроля оказалась в 2 раза выше при старте терапии сразу с комбинации. Вероятность назначения третьего препарата также была ниже в группе стартовой комбинации. Эта вероятность оценивалась, когда обе группы находились на комби- 
нированной терапии, что может говорить в пользу того, что феномен метаболической памяти развивается и фиксируется за гораздо меньшее время, чем принято было считать ранее.

Интересна популяция пациентов исследования: это пациенты с впервые установленным диагнозом, с относительно низкими показателями гликемии, без сердечно-сосудистых событий в анамнезе и с минимальными факторами риска сердечно-сосудистых заболеваний, которые, как правило, не рассматривались для участия в исследованиях по оценке сердечно-сосудистых исходов, а также отличались от популяции пациентов, принимавших участие в исследованиях ранней интенсификации терапии, таких как UKPDS и ADOPT. На самом деле эта популяция относительно молодых пациентов, с незначительными клиническими проявлениями СД 2 типа и сопутствующих заболеваний, нуждается в особом внимании, т.к. это менее исследованная группа в РКИ, которая, к тому же, менее активно обращается за медицинской помощью, считая себя относительно здоровыми. В то же время правильная стратегия лечения таких пациентов с самого начала может стать фундаментом их дальнейшего благополучия. Российские алгоритмы оказания специализированной помощи больным сахарным диабетом рекомендуют комбинированную сахароснижающую терапию на старте лечения при уровне $\mathrm{HbA}$ с выше целевого на $1 \%$. Исследование VERIFY дает клиническое обоснование для назначения комбинированной терапии сразу после постановки диагноза СД2, даже если исходный уровень $\mathrm{HbA}_{1 с}$ не превышает целевой, после комплексной оценки сопутствующих состояний, в частности, наличия множественных факторов сердечно-сосудисто- го риска и сердечно-сосудистых событий в анамнезе. Такой подход дает возможность реализации патофизиологической синергии действия двух исследованных компонентов терапии (вилдаглиптин и метформин), что потенциально может замедлить прогрессирование заболевания и развития осложнений за счет формирования положительной метаболической памяти.

\section{ЗАКЛЮЧЕНИЕ}

Тщательный гликемический контроль с момента постановки диагноза СД2 имеет важное значение для дальнейшего управления заболеванием и замедления прогрессирования осложнений. Комбинированная терапия на старте лечения СД2 является оправданной с точки зрения патофизиологии заболевания, а также имеет преимущества с точки зрения эффективности. Устойчивый длительный гликемический контроль, наблюдавшийся на фоне ранней комбинированной терапии в исследовании VERIFY, подтверждает целесообразность применения этой стратегии лечения в клинической практике. При подготовке нового издания «Алгоритмов специализированной медицинской помощи больным сахарным диабетом» может быть рекомендовано внесение положения о том, что комбинация иДПП-4 (вилдаглиптин) с метформином может быть назначена на старте терапии пациентов с СД2 сразу после постановки диагноза, даже если исходный уровень $\mathrm{HbA}_{1 с}$ не превышает целевой, после комплексной оценки сопутствующих состояний, в частности, наличия множественных факторов риска неблагоприятных сердечно-сосудистых событий и сердечно-сосудистых событий в анамнезе.

\section{СПИСОК ЛИТЕРАТУРЫ | REFERENCES}

1. Schwartz SS, Epstein S, Corkey BE, et al. The Time Is Right for a New Classification System for Diabetes: Rationale and Implications of the B-Cell-Centric Classification Schema. Diabetes Care. 2016;39(2):179-186. doi: https://doi.org/10.2337/dc15-1585

2. DeFronzo RA, Eldor R, Abdul-Ghani M. Pathophysiologic Approach to Therapy in Patients With Newly Diagnosed Type 2 Diabetes. Diabetes Care. 2013;36(Supplement_2):S127-S138. doi: https://doi.org/10.2337/dcS13-2011

3. Nauck M, Stöckmann F, Ebert R, et al. Reduced incretin effect in Type 2 (non-insulin-dependent) diabetes. Diabetologia. 1986;29(1):46-52. doi: https://doi.org/10.1007/BF02427280

4. Nauck MA, Meier JJ. The incretin effect in healthy individuals and those with type 2 diabetes: physiology, pathophysiology, and response to therapeutic interventions. Lancet Diabetes Endocrinol. 2016;4(6):525-536. doi: https://doi.org/10.1016/S2213-8587(15)00482-9

5. Mari A, Sallas WM, He YL, et al. Vildagliptin, a dipeptidyl peptidaseIV inhibitor, improves model-assessed b-cell function in patients with type 2 diabetes. J Clin Endocrinol Metab. 2005;90(8):4888-4894. doi: https://doi.org/10.1210/jc.2004-2460

6. Mari A, Scherbaum WA, Nilsson PM, et al. Characterization of the influence of vildagliptin on model-assessed b-cell function in patients with type 2 diabetes and mild hyperglycemia. J Clin Endocrinol Metab. 2008;93(1):103-109. doi: https://doi.org/10.1210/jc.2007-1639

7. Rosenstock J, Foley JE, Rendell M, et al. Effects of the dipeptidyl peptidase-IV inhibitor vildagliptin on incretin hormones, islet function, and postprandial glycemia in subjects with impaired glucose tolerance. Diabetes Care. 2008;31(1):30-35. doi: https://doi.org/10.2337/dc07-1616
8. Scherbaum WA, Schweizer A, Mari A, et al. Evidence that vildagliptin attenuates deterioration of glycaemic control during 2-year treatment of patients with type 2 diabetes and mild hyperglycaemia. Diabetes, Obes Metab. 2008;10(11):1114-1124. doi: https://doi.org/10.1111/j.1463-1326.2008.00875.x

9. He Y-L, Wang Y, Bullock JM, et al. Pharmacodynamics of Vildagliptin in Patients With Type 2 Diabetes During OGTT. J Clin Pharmacol. 2007;47(5):633-641. doi: https://doi. org/10.1177/0091270006299137

10. Vardarli I, Arndt E, Deacon CF, et al. Effects of Sitagliptin and Metformin Treatment on Incretin Hormone and Insulin Secretory Responses to Oral and \&quot; Isoglycemic\&quot; Intravenous Glucose. Diabetes. 2014;63(2):663-674. doi: https://doi.org/10.2337/db13-0805

11. Saisho Y. How Can We Develop More Effective Strategies for Type 2 Diabetes Mellitus Prevention? A Paradigm Shift from a GlucoseCentric to a Beta Cell-Centric Concept of Diabetes. EMJ Diabet. 2018;6(1):46-52

12. UK Prospective Diabetes Study (UKPDS) Group. Intensive bloodglucose control with sulphonylureas or insulin compared with conventional treatment and risk of complications in patients with type 2 diabetes (UKPDS 33). Lancet. 1998;352(9131):837-853.

13. Holman RR, Paul SK, Bethel MA, et al. 10-Year Follow-up of Intensive Glucose Control in Type 2 Diabetes. N Eng/ J Med. 2008;359(15):1577-1589. doi: https://doi.org/10.1056/NEJMoa0806470

14. Hoerger TJ, Segel JE, Gregg EW, et al. Is Glycemic Control Improving in U.S. Adults? Diabetes Care. 2008;31(1):81-86. doi: https://doi.org/10.2337/dc07-1572 
15. Laiteerapong N, Karter AJ, Moffet HH, et al. Ten-year hemoglobin A1c trajectories and outcomes in type 2 diabetes mellitus: The Diabetes \&amp; Aging Study. J Diabetes Complications. 2017;31(1):94-100. doi: https://doi.org/10.1016/j.jdiacomp.2016.07.023

16. Laiteerapong N, Ham SA, Gao Y, et al. The Legacy Effect in Type 2 Diabetes: Impact of Early Glycemic Control on Future Complications (The Diabetes \&amp; Aging Study). Diabetes Care. 2019;42(3):416-426. doi: https://doi.org/10.2337/dc17-1144

17. Phung OJ, Sobieraj DM, Engel SS, et al. Early combination therapy for the treatment of type 2 diabetes mellitus: systematic review and meta-analysis. Diabetes, Obes Metab. 2014;16(5):410-417. doi: https://doi.org/10.1111/dom.12233

18. Matthews DR, Paldánius PM, Proot P, et al. Glycaemic durability of an early combination therapy with vildagliptin and metformin versus sequential metformin monotherapy in newly diagnosed type 2 diabetes (VERIFY): a 5-year, multicentre, randomised, double-blind trial. Lancet. 2019;394(10208):1519-1529. doi: https://doi.org/10.1016/S0140-6736(19)32131-2

\section{ИНФОРМАЦИЯ ОБ АВТОРАХ [AUTHORS INFO]}

*Шестакова Марина Владимировна, д.м.н., проф., академик PAH [Marina V. Shestakova, MD, PhD, Professor]; адрес: 117036, Москва, ул. Дм. Ульянова, д. 11 [address: 11 Dm. Ulyanova str., Moscow, 117036 Russian Federation]; ORCID: https://orcid.org/0000-0002-5057-127X; eLibrary SPIN: 7584-7015; e-mail: nephro@endocrincentr.ru

Анциферов Михаил Борисович, д.м.н., профессор [Mikhail B.Antsiferov, MD, PhD, Professor]; ORCID: https://orcid.org/000-0002-9944-2997; eLibrary SPIN: 1035-4773; e-mail: antsiferov@rambler.ru Аметов Александр Сергеевич, д.м.н., професcop [Alexander S. Ametov, MD, PhD, Professor]; ORCID: https://orcid.org/0000-0002-7936-7619; eLibrary SPIN: 9511-1413; e-mail: alexander.ametov@gmail.com Галстян Гагик Радикович, Д.М.Н., професcop [Gagik R. Galstyan, MD, PhD, Professor]; ORCID: https://orcid.org/0000-0001-6581-4521; eLibrary SPIN: 9815-7509; e-mail: galstyangagik964@gmail.com. Демидова Татьяна Юльевна, д.М.н., профессор [Tatiana Y. Demidova, MD, PhD, Professor]; Researcher ID: D-3425-2018; Author ID: 7003771623; eLibrary SPIN: 9600-9796; e-mail: t.y.demidova@gmail.com

Зилов Алексей Вадимович, К.M.H. [Alexey V. Zilov, MD, PhD]; ORCID: https://orcid.org/0000-0003-3494-8011; eLibrary SPIN: 8575-1247; e-mail: azilov@hotmail.com.

Маркова Татьяна Николаевна, д.м.н., профессор [Tatyana N. Markova, MD, PhD, Professor];

ORCID: https://orcid.org/0000-0002-4882-8494; eLibrary SPIN: 5914-2890; e-mail: markovatn18@yandex.ru.

Петунина Нина Александровна, д.М.Н., професcop [Nina A. Petunina, MD, PhD, Professor];

ORCID: https://orcid.org/0000-0001-9390-1200; eLibrary SPIN: 9784-3616; e-mail: napetunina@mail.ru

Черникова Наталья Альбертовна, к.м.н., доцент [Natalya A. Chernikova, MD, PhD, associate professor]; ORCID: https://orcid.org/0000-0002-0562-8396; eLibrary SPIN: 5043-5759; e-mail: nachendoc@yandex.ru

Шамхалова Минара Шамхаловна, д.м.н. [Minara S. Shamhalova, MD, PhD];

ORCID: https://orcid.org/0000-0002-3433-0142; eLibrary SPIN: 4942-5481; e-mail: shamkhalova@mail.ru

\section{ЦИТИРОВАТЬ:}

Шестакова М.В., Анциферов М.Б., Аметов А.С., Галстян Г.Р., Демидова Т.Ю., Зилова А.В., Маркова Т.Н., Петунина Н.А., Черникова Н.А., Шамхалова М.Ш. Какие новые возможности для клинической практики открывает исследование VERIFY и какова его ценность для пациентов с впервые выявленным СД 2 типа? Совместное заключение по итогам экспертного совета. 6 ноября 2019 г. // Сахарный диабет. — 2020. — T. 23. — №1. — C. 106-110. doi: 10.14341/DM12404

\section{TO CITE THIS ARTICLE:}

Shestakova MV, Antsiferov MB, Ametov AS, Galstyan GR, Demidova TY, Zilov AV, Markova TN, Petunina NA, Chernikova NA, Shamkhalova MS. What are new opportunities for clinical practice the VERIFY study opens and which values for native diabetes patients? Joint conclusion on the advisory board results. November 6, 2019. Diabetes Mellitus. 2020;23(1):106-110. doi: 10.14341/DM12404 